Revista Estudios, (40), 2020.

Junio 2020-Noviembre 2020

ISSN 1659-3316

Cerino Córdova Kristian Antonio

Dossier: La escritura en los desplazamientos: viajes, expediciones y naufragios

\title{
El viaje en la obra narrativa del escritor Álvaro Mutis
}

Kristian Antonio Cerino Córdova

Universidad Veracruzana, México

librodemar@gmail.com

https://orcid.org/0000-0003-4306-9203

Recibido: 14 de febrero de 2020

Aceptado: 30 de marzo de 2020

Resumen: A través de las categorías de Diario y Viaje se hace un análisis de dos obras narrativas de Álvaro Mutis: La Nieve del Almirante y Un bel morir. En ambas novelas está presente el Viaje al interior: Del río, en el primer caso, y de la cordillera, en el segundo. Se trata de dos desplazamientos hechos por Maqroll el Gaviero: uno fluvial y otro terrestre.

Palabras clave: Viaje; diario; desplazamiento; tránsito

The journey in the narrative work of the writer Álvaro Mutis

Abstract: Through the categories of Diary and Travel, an analysis is made of two narrative works by Álvaro Mutis. La Nieve del Almirante and Un bel morir. In both novels the Journey to the interior is present: Of the river, in the first case, and of the mountain range, in the second. These are two movements made by Marqroll el Gaviero: one by river and the other by land

Key words: Travel; diary; displacement; transit

\section{(C) $(0 \bigcirc$}

La Revista Estudios es editada por la Universidad de Costa Rica y se distribuye bajo una Licencia Creative Commons Atribución-NoComercial-CompartirIgual 3.0 Costa Rica. Para más información envíe un mensaje a 
Dossier: La escritura en los desplazamientos: viajes, expediciones y naufragios

\section{Viajar por agua}

La Nieve del Almirante (1986) y Un bel morir (1989), obras literarias de Álvaro Mutis, están reunidas en el serial Empresas y tribulaciones de Maqroll el Gaviero. Ambas novelas, publicadas con tres años de diferencia, representan un viaje al interior de cordilleras, páramos y selvas. En la primera obra está presente el desplazamiento de Maqroll río arriba, usando un lanchón, en la búsqueda de unos aserraderos. En la segunda, el desplazamiento es hecho en recuas de mulas entre el puerto y la montaña. Son dos movimientos: uno fluvial y otro terrestre, y en los dos movimientos Maqroll el Gaviero es el protagonista. En La Nieve del Almirante él escribe, en el "Diario del Gaviero", la travesía por el río Xurandó. No hay un narrador externo porque Maqroll -como narrador- redacta el cuaderno, bitácora o diario, en primera persona, pero en Un bel morir sí está la moderación de un narrador omnisciente que nos da cuenta de los días del Gaviero en tierra adentro, en tierra caliente, como la de Golfito, en Costa Rica.

Ya sea en La Nieve del Almirante o en Un bel morir, las nociones de desplazamiento, errancia y tránsito, se perciben en ambos viajes: en el viaje-río y en el viaje-tierra. En ambos casos, el final del viaje o de la travesía se traduce en un espacio de descomposición y muerte. Estamos ante la configuración de un lugar "ambiguo, rebelde y traicionero que amenaza, que corrompe todo intento de civilización y de orden". (Raffetto, 2011, p.17)

Maqroll en sí mismo es una caravana, una caravana que al llegar a un destino no se queda en el sitio porque vuelve a emprender un camino. El Gaviero está hecho para mantenerse en constante tránsito; no permanece en la selva y mucho menos en las montañas, en La Nieve del Almirante; menos lo hace en el puerto, en los cafetales y en la cuchilla del Tambo en Un bel morir.

En los dos escenarios: tanto en el Xurandó (La Nieve del Almirante) como en Puerto Plata (Un bel morir), Maqroll ya ha renunciado a sus grandes desplazamientos de

La Revista Estudios es editada por la Universidad de Costa Rica y se distribuye bajo una Licencia Creative Commons Atribución-NoComercial-CompartirIgual 3.0 Costa Rica. Para más información envíe un mensaje a revistaestudios.eeg@ucr.ac.cr. 
Dossier: La escritura en los desplazamientos: viajes, expediciones y naufragios mar: de golfo a ensenada, de recodo a bahía, de península a cabo, de muelle a otros puertos. Aquellos viajes largos con su amigo Abdul Bashur han pasado a la historia. Esta vez, el viaje es en otra ruta o dirección: al interior. Sin embargo, atravesar la selva por medio del río y alcanzar la cúspide de la cordillera, son lugares que ocasionan tormento en la vida del Gaviero.

En La Nieve del Almirante como en Un bel morir se observan dos similitudes que podrían ser una característica de estos viajes al interior: la justicia pronta y expedida, al margen de procesos judiciales. En el contexto de ambos viajes la milicia pone fin a los intentos de grupos de criminales por trabajar en el lugar, lugares de los que ya hemos dicho están en tierra adentro; alejados de las grandes ciudades, entre el río y el monte, aquí todo es campo abierto para el enfrentamiento con armas, el tráfico de personas y armamento, y la gran posibilidad de transitar en la ilegalidad.

La Nieve del Almirante no solamente es un Diario de viaje, sino un viaje doméstico, a lo profundo de la casa o de la tierra. Esta obra narrativa de Mutis (ocurre también con Un bel morir) es, además, una travesía por un mundo hostil que es visto a través de una mirada desencantada, la de Maqroll, autor del "Diario del Gaviero". Cuando Maqroll va escribiendo el diario, al mismo tiempo en que viaja en el lanchón, este mismo diario se transforma en el fluir de una existencia que transcurre en la orilla. Si bien el Diario de viaje ya no hace registros de aquellos lugares inhóspitos, hoy permite o posibilita habitar provisionalmente el único lugar y tiempo posible: el de la ficción. La escritura en el diario no es más que un movimiento, un caminar de nuevo y de forma diferente el camino. Así, Maqroll emprende el viaje al interior en dos sentidos: el desplazamiento río adentro y el viaje que nos muestra el Yo del Gaviero a través de sueños y lecturas. En Un bel morir ya no está presente la forma Diario (el registro-bitácora), pero se mantiene el espíritu del viaje como un desplazamiento al centro de la tierra (pensemos en Verne). La idea es caminar, subir a la cúspide, y volver al muelle. Es como un viaje circular con un propósito: mirar lo que está en aquellos senderos por donde se transita poco, mirar los detalles, mirar los adentros.

\section{(c) (i) (-)}

La Revista Estudios es editada por la Universidad de Costa Rica y se distribuye bajo una Licencia Creative Commons Atribución-NoComercial-CompartirIgual 3.0 Costa Rica. Para más información envíe un mensaje a revistaestudios.eeg@ucr.ac.cr. 
Dossier: La escritura en los desplazamientos: viajes, expediciones y naufragios

El escritor mexicano Sergio Pitol publicó El viaje (2000), crónica y Diario de viaje para conducir al lector por los caminos de algunas ciudades soviéticas, entre ellas, Tiflis, capital de Georgia. Pitol, el viajero que abordó barcos, trenes y aviones, escribe a través de una serie de referencias culturales y para ello recurre a su Cuaderno de viaje que es el soporte del libro. Éste es más que una cartografía de viaje: es la suma de experiencias, de lecturas y de visiones que Pitol, narrador mexicano que vivió más de treinta años en el extranjero, proyecta en El viaje muchos años después de esta travesía por los rincones soviéticos. A esta publicación le antecedió El arte de la fuga, primera obra de la "Trilogía de la memoria" en la que narra dos décadas de sus experiencias como escritor, lector, traductor y viajero. Para Fernández de Alba (2005) Pitol empleó en su obra narrativa y ensayística el viaje como protagonista. En otras palabras, el viaje como categoría. En Pitol está presente el viaje iniciático, el viaje como tregua, el viaje como refugio interior, la traducción literaria como viaje literario, el viaje como evasión, el viaje como actividad continua y el viaje como elemento literario. En Álvaro Mutis están los rasgos del Viaje al interior.

"Pero las interesantes crónicas de sus días de viaje por Moscú, Leningrado y, finalmente, Tbilisi, se irrumpen en varias ocasiones para dar paso a diferentes textos que, intercalados, completan la visión del viajero" (Fernández de Alba, 2005, p.557)

La obra mutisiana, estudiada un sinfín de veces a través de las categorías de Deterioro, Desesperanza, Alter Ego, entre otras, merece atención aparte una revisión en otro sentido; en categorías como Diario y Viaje, el viaje visto por encima de una simple temática, pues éste también debe ser abordado como protagonista.

El viaje al interior es "un tema difícil pues presenta un tipo particular de dilema discursivo para el viajero regional, relativo precisamente a las herramientas retóricas y las estrategias empleadas en la construcción de la narrativa de viaje". (Lindsay, 2016, p.31-32).

\section{(c) (i) (-)}

La Revista Estudios es editada por la Universidad de Costa Rica y se distribuye bajo una Licencia Creative Commons Atribución-NoComercial-CompartirIgual 3.0 Costa Rica. Para más información envíe un mensaje a revistaestudios.eeg@ucr.ac.cr. 
Dossier: La escritura en los desplazamientos: viajes, expediciones y naufragios La Nieve del Almirante está organizada esencialmente por el "Diario del Gaviero", un relato hecho por Maqroll, el marinero errante. Grosso modo es un Diario de Viaje por el interior. Esta composición genérica en la obra de Álvaro Mutis ha requerido de un estudio en el que se exploran los múltiples géneros que hay en la redacción del "Diario del Gaviero"; entre ellos, la carta y la letanía. Se observa, en un principio, que La nieve del Almirante está tejida, en su estructura, alrededor del Diario: un género que germinó en el medievo, que se leyó con interés en el siglo XIX y que se sobreexplotó en el XX.

Bajtín afirma que hay un grupo especial de géneros que tiene un rol esencial en la construcción de la novela, y que, en ocasiones determina la estructura del conjunto de la novela, estableciendo así los cambios especiales del género novelesco. En el grupo están: la confesión, el diario íntimo, el diario de viajes, la biografía y la carta. "Todos ellos, no sólo pueden entrar en la novela como parte constitutiva fundamental, sino determinar también la forma de la novela como un todo (novelaconfesión, novela-diario, novela-epistolar, etc.)". (Bajtín, 2011, p.138).

El diario, en La Nieve del Almirante, es, además de un engrane, la forma que posibilita el orden de la escritura y la correspondencia entre la unidad-diario y las otras formas literarias que están en el relato.

Ahora bien, cuando Maqroll está escribiendo el "Diario del Gaviero" lo hace "por turnos" (p.78), lo redacta en las madrugadas mientras la tripulación del navío duerme, y para hacerlo, debe alumbrarse con "la lámpara" Coleman (p.155), de las usadas en la minería. En resumen: el marinero escribe para "distraerse" (p.152) ante la espera por conocer los aserraderos en una de las márgenes del río Xurandó.

En la redacción del "Diario del Gaviero" Maqroll trabaja a la par en otros procesos de escritura, pero como parte del mismo diario. Hace uso de la transcripción al reproducir la oración "para los caminantes en peligro de muerte" (p.107) que ha hecho el capitán del lanchón y que la comparte con Maqroll en el instante en que ingresan a los rápidos del afluente.

\section{(C) $(\otimes \odot \bigcirc$}

La Revista Estudios es editada por la Universidad de Costa Rica y se distribuye bajo una Licencia Creative Commons Atribución-NoComercial-CompartirIgual 3.0 Costa Rica. Para más información envíe un mensaje a 
Dossier: La escritura en los desplazamientos: viajes, expediciones y naufragios

Esta recapitulación del proceso escritural en Maqroll es pertinente porque todo lo escrito -citado o referenciado en La Nieve del Almirante- permite comprender su capacidad narrativa para afrontar el relato en el "Diario del Gaviero".

El viaje también es visto como el acto de hacer una mudanza de los sentidos y es "una experiencia antropológica que ha marcado diferentes caminos en la estética y en la hermenéutica" y "su diversidad les aporta características y funciones históricas bien distintas, pero su origen común les otorga una línea de parentesco que en los tiempos modernos les permite reestablecer vínculos asociativos y reencuentros (Beltrán, 2011, p. 112). Así, viaje y narración van juntos "por su divagante condición de desplazamiento y de intrigado azar, que se declina encarnándose en los semblantes de la prueba, la iniciación, la exploración, el conocimiento, el peregrinaje, el esparcimiento, la huida (Manera, 2007, p. 166).

Maqroll escribió el "Diario del Gaviero" entre el 15 de marzo y el 29 de junio. No sabemos en qué año lo hizo y se desconoce en qué año fue hallado este manuscrito en Barcelona. El Diario, escrito por Maqroll durante lo más de setenta y cuatro días de travesía por el río en la búsqueda de los aserraderos, es el soporte del registro hecho en las treinta y tres "entradas" redactadas por este navegante, en este su Viaje al interior.

\section{Viajar por tierra}

Un bel morir (1989) no sólo es un viaje por los caminos sinuosos del páramo. Es, además, un viaje tierra adentro para mostrarnos la vida y sus vericuetos allá por donde caminan unos cuantos. Un bel morir es también el desplazamiento al interior como un destino a través de la mirada de los personajes que habitan en esta novela de Álvaro Mutis y que pertenece a la saga de "Maqroll el Gaviero".

Esta vez, así como en La Nieve del Almirante (1986), Maqroll el Gaviero es el protagonista del viaje cuyo punto de partida es Puerto Plata y cuyo punto de llegada

\section{(cc) (i) ()}

La Revista Estudios es editada por la Universidad de Costa Rica y se distribuye bajo una Licencia Creative Commons Atribución-NoComercial-CompartirIgual 3.0 Costa Rica. Para más información envíe un mensaje a 
Dossier: La escritura en los desplazamientos: viajes, expediciones y naufragios es la Cuchilla del Tambo. A diferencia de sus días navegando el río Xurandó en la búsqueda de los aserraderos, en esta ocasión, el marinero decide dejar las rutas fluviales para desplazarse a pie, en mulas y a caballo. Al desembarcar del lanchón, el Gaviero ha emprendido otro viaje a ras de tierra como una caravana inagotable, siempre en constante movimiento.

En esta narración en tercera persona, ya no es la voz que escuchamos por medio del "Diario del Gaviero" en La Nieve del Almirante, sino la de un narrador omnisciente que cuenta los días del navegante en el llano, en el páramo, en la cuchilla. Maqroll se ha apartado de la escritura como se apartó del lanchón que lo hizo llegar a Puerto Plata, para así montarse en animales de carga.

A diferencia de la novela La Nieve del Almirante que está construida alrededor del Diario por otros géneros literarios, Un bel morir está hecha con base en otra forma: es una narración lineal con algunas analepsis que nos dan pista de la esencia del Gaviero. Podría decirse que estamos frente a un doble viaje: entre el puerto y la cuchilla -elevación montañosa- y entre el ser de Maqroll. Sin embargo, ambas obras literarias están comunicadas por el Viaje, un viaje sí guiado por el mismo protagonista, del marino que cada vez se aleja del mar, del puerto, del muelle, del agua.

El viaje de Puerto Plata a la cuchilla del Tambo requiere de seis días; tres para subir, tres para bajar. Tres viajes son los que realiza Maqroll el Gaviero para transportar cajas de madera en cuyo interior, se sabrá después, hay armas que están destinadas para grupos de criminales que se apoderan del monte, en lo alto de la cordillera, y desplazan a las familias de campesinos.

Este camino de Maqroll ya no es el serpenteo del río que fluye entre rápidos y roquedales. El río que se arrastra al pasar entre líneas curvas y rectas para fusionarse con la mar, ha quedado atrás, por lo pronto, en el itinerario del Gaviero. Ahora, este serpenteo tiene otra forma en Un bel morir, en el trayecto de subida al

\section{(c) (i) (2)}

La Revista Estudios es editada por la Universidad de Costa Rica y se distribuye bajo una Licencia Creative Commons Atribución-NoComercial-CompartirIgual 3.0 Costa Rica. Para más información envíe un mensaje a revistaestudios.eeg@ucr.ac.cr. 
Dossier: La escritura en los desplazamientos: viajes, expediciones y naufragios páramo que huele a tierra mojada, a cafetales y a un aire puro que ahoga, en donde igualmente se olfatea una gran peligrosidad. Es quizá la percepción de la muerte lo que está presente en el camino de tierra en Un bel morir como antes lo estuvo en el camino de río en La Nieve del Almirante. El instante en que Maqroll inicia este camino a pie es cuando llega a Puerto Plata al ya no tener noticias de los amigos. Se ha quedado anclado o fondeado como navío en este puerto y pueblo caluroso, y de pocos caseríos. El anclaje del Gaviero es brevísimo porque luego emprende el camino de tierra en los Tres viajes a la cuchilla del Tambo.

El mismo epígrafe de la obra Un bel morir dice: "Y a pas de port" (No hay ningún puerto). La cita es del poeta francés Jules Laforgue, quien previamente en el mismo poema Sole de Lune, escribe: "Tout n'en va pas moins à la Mort" (Nada se mueve salvo a la muerte). Maqroll se halla fondeado, por unos días, en Puerto Plata. Sin embargo, su destino no está allí. Por ello, es pertinente que comience un nuevo camino, ese Viaje al interior de un lugar o al camino-destino que ya le depara.

Realice el viaje a través del río o por los caminos angostos de la cordillera, uno de los propósitos de Maqroll es seguir una corriente o una vía que lo desplace de un punto de partida a otro de llegada. Esta vez, hará Tres viajes o desplazamientos circulares, de ida y vuelta, entre el abajo y el arriba de este lugar del interior de una región montañosa.

En otras ocasiones, el Gaviero había ya navegado frente a Puerto Plata. Empera, la anciana ciega que lo hospeda y protege de los vivales, asegura conocerlo -con anterioridad- porque ha escuchado de su tránsito fluvial por este río de aguas turbias.

Desde el principio en Un bel morir, Maqroll sabe, por las historias que cuenta Empera, lo peligroso de permanecer en Puerto Plata y está al tanto del riesgo que asume si emprende el viaje con mulas a la Cuchilla del Tambo. En resumen: la quietud de Puerto Plata no debe generarle un ambiente de confianza, y la

La Revista Estudios es editada por la Universidad de Costa Rica y se distribuye bajo una Licencia Creative Commons Atribución-NoComercial-CompartirIgual 3.0 Costa Rica. Para más información envíe un mensaje a revistaestudios.eeg@ucr.ac.cr. 
Dossier: La escritura en los desplazamientos: viajes, expediciones y naufragios tranquilidad de su río es sólo una apariencia porque más allá de su color lodoso, la sangre derramada de campesinos en otros tiempos por la violencia, le ha dado otra tonalidad más turbia a este afluente.

Antes de que salga la caravana de mulas guiadas por Maqroll, la vida de éste pende del destino (no es de extrañarnos porque enfrentarlo es su condición). El cuarto que renta en el hostal está hecho de bambú y está suspendido sobre la corriente del río. Desde el balcón de bambú contempla la inmensidad de la cordillera, "lo inalcanzable del páramo" (p.19), la fragilidad de la humanidad ante la majestuosidad de la montaña. El cuarto, a unos metros del puerto, le permite mirar el destino, ver hacia adentro a través de sus reflexiones y sueños; y mientras se acuesta en la cama de guadua o bambú, escucha el río que corre por debajo y que lo "ayuda a olvidar" (p.116). En sus viajes de ida y vuelta que duran seis días, el Gaviero retorna a su habitación para leer, meditar, recordar y, de paso, oír y ver el correr del río "indiferente a la milenaria torpeza de los hombres, a su desventurada vocación de sacrificio" (p.179).

¿Por qué el Gaviero se da la concesión de hacer un viaje tierra adentro? (como ir del Golfito a San José) ¿Por qué se alejó del mar y se replegó en Puerto Plata para iniciar una caravana a la Cuchilla del Tambo? ¿Por qué dejó los tránsitos fluviales para emprender otros de tipo terrestre? No lo dice con estas palabras, pero éstas se acercan a la condición de Maqroll: para experimentar "subiría, pues" a la cuchilla. (p.34). En otros fragmentos de esta obra literaria, el Gaviero está consciente que siempre se embarca en empresas que descansan "en el aire". Ahora no será la excepción. Subiría a lo alto de la colina, acompañado del joven Zuro, para llevar las cajas de madera, porque su destino no está al margen de la historia, sino en el centro de ésta. Maqroll es el personaje elegido para llegar al lugar-destino. Estando aquí escuchará, mirará y experimentará para luego partir en la búsqueda de algo más que aún no encuentra. A decir de Maqroll siempre que intenta hacer algo en tierra adentro "(le) va mal" (p.133). No es el único que piensa así. Aníbal, el

\section{(c) (†)(2)}

La Revista Estudios es editada por la Universidad de Costa Rica y se distribuye bajo una Licencia Creative Commons Atribución-NoComercial-CompartirIgual 3.0 Costa Rica. Para más información envíe un mensaje a 
Dossier: La escritura en los desplazamientos: viajes, expediciones y naufragios campesino que lo asiste con mulas y provisiones en el Llano de los Álvarez, hace uso de la filosofía cotidiana para decirle al Gaviero en dónde podría estar la solución a todos sus males: "Busque el mar, allí está su salvación" (p.132). Con anticipación el mismo Aníbal había dicho que "el río es el que trae las sorpresas más terribles". (p.100), y Empera, la ciega solidaria en Puerto Plata, a su vez, afirmaba que "la vida es como estas aguas de río que todo lo acaban nivelando, lo que traen y lo que dejan, hasta llegar al mar", (porque) la corriente es "siempre la misma". (p.88).

Tres viajes realizan Maqroll a la Cuchilla del Tambo. Dieciocho días dedica a la misión encargada por un falso belga y contrabandista, que lo contrata para subir en recuas de mulas cajas de maderas que contienen armamento. Así como el río Xurandó que está a merced de los bribones y bandidos, así también lo está el camino entre Puerto Plata y el Tambo. En el Xurandó los soldados se desplazan río abajo y río arriba, y por aire en un hidroavión, para desaparecer a los contrabandistas. En la subida a la Cuchilla del Tambo, Maqroll recuerda aquella “justicia expedita” del ejército en el Xurandó (p.82) y la ocasión en que pudo morir ahogado en los rápidos o enfermo de malaria.

Un escenario semejante ocurre en Puerto Plata, en el Llano de los Álvarez, la cabaña abandonada de los Mineros y en la Cuchilla del Tambo; éste el lugar final de la travesía y el sitio que eligieron los seudo constructores de las vías ferroviarias para acumular armas en bodegas y de esta manera orquestar una guerrilla. El resultado, sabremos más tarde, fue el aniquilamiento de los rebeldes.

Desde los primeros momentos de Un bel morir, el narrador describe la presencia de un puesto militar en Puerto Plata y deja constancia de la violencia en este lugar del interior; una violencia de "un sombrío pronóstico indefinible", según palabras de Empera, la dueña del hostal a orillas del río (p.21). No debemos olvidar que los habitantes del Llano de los Álvarez llegaron de otras zonas apartadas, precisamente "huyendo de la persecución política" (p.39). Todo este contexto lo tiene presente el

\section{(c) (i) (-)}

La Revista Estudios es editada por la Universidad de Costa Rica y se distribuye bajo una Licencia Creative Commons Atribución-NoComercial-CompartirIgual 3.0 Costa Rica. Para más información envíe un mensaje a revistaestudios.eeg@ucr.ac.cr. 
Dossier: La escritura en los desplazamientos: viajes, expediciones y naufragios Gaviero. Es más, sospecha que la misión o el encargo del "belga" es algo turbio, pero la condición humana de Maqroll lo conduce a un objetivo: debe llegar a su destino y debe experimentar la travesía. No tendría sentido emprender una caravana para dejarla varada a mitad del camino.

El primer viaje por tierra es la prueba iniciática del Gaviero. Ha dejado atrás un río "traicionero" y se perfila a subir una "cuchilla traicionera", de acuerdo con los adjetivos que emplea el narrador al ir marcando el tiempo de la narración con pautas como éstas: "Al día siguiente" (p.45, 78), "Cuando llegó la noche" (p.52), "A la madrugada siguiente" (p.56)....Esta prueba inicial de Maqroll era difícil emprenderla sin la ayuda de un conocedor de la Cuchilla del Tambo: el Zuro, una especie de escudero cervantino que va abriendo brecha durante la subida. Él es más que un personaje secundario: es vital para concluir el ascenso. El Zuro es un lobo alfa guiando a la caravana, a las recuas, al mismo Gaviero. Con los ojos de él, Maqroll puede ver y anticiparse a lo que vendrá. Siempre adelante como quilla de barco: partiendo y abriendo el camino. En la medida que suben, Zuro dice una sentencia al descubrir que no hay rastros de construcción de vías férreas, si recordamos que lo que transportan en las cajas son herramientas de la supuesta empresa ferroviaria: "Hay que andarse con cuidado, mi don. No sé por qué se me ocurre que, al final, lo van a querer joder" (p.74). Aníbal dirá después que una carretera suple ya la función que tendría el ferrocarril (p.80). Así, avanzan entre caminos pedregosos, lodosos, arcillosos. Las cinco mulas que conforman las recuas se hunden, se atascan entre "la arena volcánica" (p.69) sin que el Gaviero escuche el "tintineo" de los metales entre las cajas que acarrea. El viento es fuerte mientras está el ascenso. Se trata del primer viaje y ya el responsable de la misión está pensando seriamente en abortar. Si en los ojos de las bestias de carga hay "pánico" (p.76), en los de Maqroll hay más. En este primer viaje, el Gaviero se hace una idea de la posible conspiración ante la supuesta ausencia de gobierno.

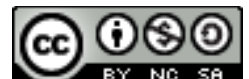

La Revista Estudios es editada por la Universidad de Costa Rica y se distribuye bajo una Licencia Creative Commons Atribución-NoComercial-CompartirIgual 3.0 Costa Rica. Para más información envíe un mensaje a revistaestudios.eeg@ucr.ac.cr. 
Dossier: La escritura en los desplazamientos: viajes, expediciones y naufragios Entre un viaje y otro hay una espera por múltiples factores: el cansancio de las mulas, la llegada de más cajas con armas, el dinero para costear el ascenso, la sospecha de lo ilegal, el clima. ¿Cuál es la diferencia entre el primer viaje y el segundo? Podría ser la pérdida de voluntad, de fuerzas, de mulas. En este segundo viaje, Maqroll confirma su sospecha de caminar sobre un suelo de revueltas, se da cuenta que ha sido usado para transportar armas. Su observación y las conjeturas de Zuro le permiten hacer nuevas conclusiones sobre lo que está ocurriendo en el monte. Para entonces, ya sabe que los dichosos belgas (un alemán y otro holandés) llegaron a Puerto Plata ante la ausencia de milicianos. En la zona de mayor inclinación Maqroll teme caer, estrellarse contra las rocas del lejanísimo río. El miedo del Gaviero salta con "felina regularidad" (p.110). Estos temores, es probable, estén presentes en las recuas que una de las mulas cae al precipicio. Pese a la caída de la mula cerca de Plan de Santa Ana, el viaje continúa en estos caminos en zigzag (p.93). Atrás, pero muy atrás, quedó el serpenteo del agua. Lo que se mantiene aquí es el serpenteo del camino, el zigzag de la vía que cada vez se hace angosta. Llueve, truena, relampaguea. Las luces iluminan "los ojos desorbitados" de las mulas (p.103). Llegan a la cabaña de los Mineros para guarecerse de la lluvia y seguir comiendo plátano con café; dormir sobre hojas de frailejón y atender a las mulas que yacen cansadas en el establo. "Los belgas" le recriminan por la pérdida de una mula por lo que es necesario ir por sus restos y por la caja de madera. El segundo viaje está plagado de pausas como éstas: es necesario salirse de la ruta, hallar los restos, llegar antes que otros, repasar el plan de la caravana, cuidar las recuas que están nerviosas al pasar por un camino angosto, y estar alerta de las asechanzas. Al menos el Gaviero goza de la ventaja que le da el Zuro, conocedor de estos caminos angostos. Deben volver y descender hasta el río (otra vez al río). Sólo hallan residuos de cajas y la etiqueta CZEC, de origen checo. De la herramienta ferroviaria poco se sabe hasta que Aníbal le informa que el contenido era una ametralladora que ya ha confiscado el ejército, que "correrá sangre", que

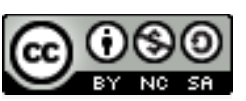

La Revista Estudios es editada por la Universidad de Costa Rica y se distribuye bajo una Licencia Creative Commons Atribución-NoComercial-CompartirIgual 3.0 Costa Rica. Para más información envíe un mensaje a revistaestudios.eeg@ucr.ac.cr. 
Dossier: La escritura en los desplazamientos: viajes, expediciones y naufragios inteligencia militar supo desde un principio de las armas y que la violencia siempre estuvo en estos lugares. De Maqroll dice que sus aventuras son para él como una lección para aprenderlas. En este segundo viaje, ya en el descenso al Llano de los Álvarez, Aníbal pide al Gaviero marcharse de Puerto Plata y ayudar al capitán Segura a transportar las últimas cajas a la Cuchilla del Tambo, con la venia del ejército y a sabiendas de que es peligroso volver a la colina plagada de contrabandistas y revoltosos. Maqroll teme volver ante la advertencia de Aníbal y de Empera, quienes pronostican que el próximo viaje "se pondrá feo". Mientras espera las nuevas cajas de madera que llegarán al muelle, se recuesta en la cama y oye el manso "murmullo" del río que pasa bajo el cuarto que renta. Sueña y estos sueños lo ayudan a olvidar "la oculta trama de su destino" (p.139). Todo se esfuma, como en los sueños, también este viaje a ras de tierra. Amparo María, otra mujer que ama al Gaviero, le anticipa que en su tercer viaje lo esperará en el camino como creyendo que estos desplazamientos son rutinarios y sin alteraciones. Idénticos. Qué poco conoce al marinero. No lo vuelve a ver.

Maqroll alista su tercer viaje. Emprende el camino con el consejo de la ciega Empera: "vaya con el ojo abierto". Así, parte con la esperanza de que al retornar la anciana le dará una noticia. Se marcha con esa idea de saber de qué se trata; se va cansado, al último viaje terrestre, y sintiendo que los años ya le pasaron encima, con evidencias de ese deterioro. Parece un Larsen (de Onetti) abatido.

En la subida se escuchan las primeras explosiones. Hay un combate entre los falsos constructores del ferrocarril y los soldados. Esto no detiene al Gaviero que sigue escalando la colina llevando las últimas dos cajas a la cabaña de los mineros y pensando en salir con vida de este su tercer viaje. Como en las subidas anteriores, en ésta no llueve con dureza, pero la lluvia -ahora- se torna de plomo. Hay tensión. No es lo mismo ir en ascenso con el miedo a desplomarse que ir en subida con el ruido de las balas. Maqroll cumple con la tarea de llevar las cajas y comienza en lo inmediato el descenso al Llano de los Álvarez. Los descensos son más rápidos pero

\section{(c) (i) (ㅇ) (2)}

La Revista Estudios es editada por la Universidad de Costa Rica y se distribuye bajo una Licencia Creative Commons Atribución-NoComercial-CompartirIgual 3.0 Costa Rica. Para más información envíe un mensaje a revistaestudios.eeg@ucr.ac.cr. 
Dossier: La escritura en los desplazamientos: viajes, expediciones y naufragios el Gaviero mantiene la calma pese a los estallidos que se oyeron en los alrededores de las bodegas y de la cabaña. Se despide de Aníbal y de Zuro, a quien llama "un compañero ejemplar". Esta será la última vez que los vea. Serán asesinados, así como Amparo María y otros más del llano. Sólo un sobreviviente dará la noticia a Empera de la masacre entre el monte. Maqroll querrá retomar su huida en un lanchón deteriorado. No podrá. Antes será arrestado por el ejército, interrogado y puesto a prueba sobre si en verdad ayudó en el último viaje al capitán Segura a desenmascarar a los rebeldes.

Un capitán de apellido Ariza le dirá días después del arresto, que su país, Chipre, ha pedido su liberación; que ya puede irse: "Ahí está su lanchón. Aquí tiene un salvoconducto para circular en nuestra zona. Ojalá le sirva" (porque) "usted estaba muerto hace rato" (p.211-212)

En las hojas finales de Un bel morir, el Gaviero abandonará Puerto Plata. Un anciano le ayudará a empujar el lanchón para que se aleje lo más pronto posible ante la advertencia de los milicianos, de no volver, que lo han puesto en libertad. Ese empujón del viejo lo pone, otra vez, a navegar pese a que él ya se siente vencido como un "Caronte" (p.220), como aquel barquero mitológico. No lleva nada consigo, solo el deseo de hallar "reposo".

Maqroll parte de Puerto Plata en el lanchón corroído. Se va con la noticia de que Flor Estévez estuvo aquí, buscándolo, amándolo. Abandona el pueblucho caluroso río arriba, quizá pensando en lo tortuoso que fue este viaje tierra adentro, al interior. Como en todo viaje circular, Maqroll vuelve a su origen: el río que da salida al mar.

\section{Acotación:}

Puntarenas, Costa Rica, está en la cartografía de la obra literaria de Álvaro Mutis. Aunque esté escrito como Punta Arenas (la ciudad y puerto de Chile), el narrador de la novela La última escala del Tramp Steamer (1988) dice que el vapor "El Alción"

\section{(c) (i) (9) (2)}

La Revista Estudios es editada por la Universidad de Costa Rica y se distribuye bajo una Licencia Creative Commons Atribución-NoComercial-CompartirIgual 3.0 Costa Rica. Para más información envíe un mensaje a revistaestudios.eeg@ucr.ac.cr. 
Dossier: La escritura en los desplazamientos: viajes, expediciones y naufragios ha llegado a la bahía de Nicoya, Costa Rica. Este carguero hondureño, agotado y deteriorado, navegará por todo el Pacífico frente a las costas de Puntarenas hasta llegar a Panamá. El barco, de acuerdo con el narrador, lo ha visto en Helsinki (Finlandia), llevando aceite de oliva; y lo verá más tarde en Kingston (Jamaica). El narrador, al estar en Puntarenas, ve también pasar este navío de "agónico estertor" en sus motores. En La última escala del Tramp Steamer Jon Iturri, el capitán del Alción, lleva mercancía de puerto en puerto ante la incredulidad de muchos por lo arruinado que está el vapor. A la par del viaje sostiene una relación amorosa con Warda Bashur, hermana de Abdul Bashur, el gran amigo de Maqroll el Gaviero: el eterno navegante. Un día, Abdul apareció en Puntarenas. Iturri y él viajan al valle de San José, Costa Rica. En esta ciudad capital Iturri suelta una sentencia: "Lo de ustedes durará lo que dure el Alción". Así fue. El narrador sabrá esto tiempo después cuando Iturri le cuente cómo perdió el amor de Warda y cómo naufragó el carguero en el río. Celebro estar en Golfito, en la región de Puntarenas. No sé con exactitud si "El Alción" hizo escala en la Herradura o en el Golfito, pero sí sé que este barco deteriorado, que navegó por el mundo, estuvo en Puntarenas. Fue visto por el narrador de La última escala del Tramp Steamer y por otros testigos, desde luego mostrando su vejez, pero sin perder el ánimo de mantenerse a flote, sólo deslizándose "irreal, con el jadeo agónico de sus máquinas".

Así como en el puerto de Veracruz (México) escribí un cuento para dar otros pormenores de que El Alción estuvo aquí para entregar un cargamento de papel, así también escribo un cuento para detallar su estancia en Golfito: probablemente para llevar aceite de palma, piña o plátano. Celebro estar en Golfito, el puerto de agua dulce de Puntarenas, en donde habré de ambientar seguramente alguna novela. Soy de Tabasco, un estado o provincia que está en el Golfo de México. Por esta razón me siento en casa aquí en Golfito. Como bien dijo el Dr. Leonardo Sancho, "no dudaste en venir a Golfito". Estoy atrapado por el calor y la lluvia del lugar, pero a gusto. Golfito, Puntarenas. Octubre 24, 2019.

\section{(요요}

La Revista Estudios es editada por la Universidad de Costa Rica y se distribuye bajo una Licencia Creative Commons Atribución-NoComercial-CompartirIgual 3.0 Costa Rica. Para más información envíe un mensaje a revistaestudios.eeg@ucr.ac.cr. 
Dossier: La escritura en los desplazamientos: viajes, expediciones y naufragios

Texto leído en el Congreso Internacional: "La escritura en el desplazamiento: viajes, expediciones y naufragios", organizado por la Cátedra Humboldt y la Universidad de Costa Rica, en San José y Golfito. Octubre 2019.

Kristian Antonio Cerino Córdova es académico y periodista. Hizo estudios de Comunicación y Docencia en la Universidad Juárez Autónoma de Tabasco. UJAT. Estudia el doctorado en Literatura Hispanoamericana en el Instituto de Investigaciones Lingüístico-Literarias, IIL-L, de la Universidad Veracruzana, México.

Ha publicado artículos y ensayos sobre Literatura, Periodismo, y Tecnologías, en revistas arbitradas. Es Editor Asociado de las revistas: "Perspectivas Docentes" y "Cinzontle, en la UJAT", y en "Diálogos", publicación del Consejo de Ciencia y Tecnología de Tabasco. E-mail: librodemar@gmail.com

\section{Referencias bibliográficas:}

Bajtín, Mijail. (1989). "La palabra en la novela” en Teoría y estética de la novela. Trad. Helena S. Kriúkova y Vicente Cazcarra. Madrid: Taurus.

Beltrán, Luis. (2011) “Novela y diario" en El diario como forma de escritura y pensamiento en el mundo contemporáneo. España: Editorial Institución "Fernando el Católico" y Universidad de Zaragoza.

(2007). "El viaje como categoría estética" en Palabras de viaje, Estética y hermenéutica del viaje. España: Ediciones Vitel-la.

Fernández del Alba. (2005). "Luz. Sergio Pitol, escritor mexicano de largas, intensas, travesías" en Homenaje a Alejandro de Humboldt. Literatura de viajes desde y hacia Latinoamérica, siglos XV-XXI. México: Humboldt State University y Universidad Autónoma "Benito Juárez" de Oaxaca.

\section{(c) (i) (-)}

La Revista Estudios es editada por la Universidad de Costa Rica y se distribuye bajo una Licencia Creative Commons Atribución-NoComercial-CompartirIgual 3.0 Costa Rica. Para más información envíe un mensaje a revistaestudios.eeg@ucr.ac.cr. 
Dossier: La escritura en los desplazamientos: viajes, expediciones y naufragios

Lindsay, Claire. (2016). Escritura contemporánea de viajes de América Latina. Bogotá: Instituto Caro y Cuervo.

Manera, Danilo. (2007). "Viajes de papel y papeles de viaje: apuntes sobre andanzas literarias" en Palabras de viaje, Estética y hermenéutica del viaje. España: Ediciones Vitel-la.

Mutis, Álvaro. (2002). La nieve del Almirante. España: Punto de lectura. (2003). Un bel morir. España: Punto de lectura.

Pitol, Sergio. (2000). El viaje. México: Editorial Era.

Raffeto, Roberta. (2011). Álvaro Mutis: hacia una épica posmoderna. Alemania: Editorial Académica Española.

Rodríguez, José Ángel. (2005). "Los paisajes interiores de Elisabeth Gross" en Homenaje a Alejandro de Humboldt. Literatura de viajes desde y hacia Latinoamérica, siglos XV-XXI. México: Humboldt State University y Universidad Autónoma "Benito Juárez" de Oaxaca. 